\title{
Persistência da Imagem Metastática Pulmonary Após Tratamento de Doença Trofoblástica Gestacional
}

\author{
Persisting Metastatic Pulmonar Imaging After Treatment \\ of Gestational Trophoblastic Disease
}

Eddie Fernando Candido Murta, Marcelo Cunha Fatureto

\begin{abstract}
RESUMO
O objetivo deste relato é a apresentação de um caso de doença trofoblástica gestacional com metástases pulmonares, cujas imagens persistiram após a normalização dos títulos de fração $\beta$ do hormônio da gonadotrofina coriônica ( $\beta$ - $h C G)$ após cinco ciclos de quimioterapia (metotrexato, $20 \mathrm{mg} /$ dia por 5 dias a cada 14 dias). A paciente foi submetida a ressecção das lesões por toracoscopia vídeo-assistida. O exame histológico demonstrou necrose sem evidência de tumor residual. É importante reconhecer que a persistência de nódulos pulmonares em pacientes com doença trofoblástica gestacional metastática após tratamento e normalização do $\beta$-hCG pode não representar tumor viável mas somente necrose e/ou fibrose.
\end{abstract}

PALAVRAS-CHAVE: Doença trofoblástica gestacional. Quimioterapia. Gonadotrofina coriônica.

Introdução

O pulmão é um dos sítios de maior freqüência de metástases de doença trofoblástica gestacional (DTG). Cerca de 45 a $87 \%$ das pacientes com diagnóstico inicial de DTG maligna apresentam focos de implantação pulmonar empregando-se a radiografia de tórax como método diagnóstico ${ }^{4,6}$. O

Disciplina de Ginecologia e Obstetrícia e Disciplina de Cirurgia Torácica da Faculdade de Medicina do Triângulo Mineiro Correspondência:

Eddie Fernando Candido Murta

Disciplina de Ginecologia e Obstetrícia

Rua Getúlio Guaritá, s/n ${ }^{\circ}$ - Abadia

38025-440 - Uberaba - MG

Telefone (034) 318-5326 - Fax (034) 333-8710

E-mail: eddiemurta@mednet.com.br uso da tomografia computadorizada (TC) aumenta a sensibilidade para detectar alterações pulmonares da DTG em relação à radiografia simples, sendo portanto o melhor método de diagnóstico e seguimento ${ }^{3}$. Excelentes resultados com a quimioterapia estão associados à cura desta enfermidade, com remissões completas dos focos de metástases pulmonares ${ }^{1}$. Não obstante, a persistência da imagem metastática pulmonar associada ao decréscimo ou negativação do $\beta$-hCG tem sido descrita ${ }^{9}$. Existem poucos relatos na literatura mundial e não é de nosso conhecimento a descrição de caso semelhante na literatura nacional. Este relato tem como objetivo apresentar o caso de uma paciente com DTG metastática para os pulmões que apresentou, após cinco ciclos de 
quimioterapia, negativação do $\beta$-hCG mas com persistência de imagem metastática pulmonar.

\section{Relato de Caso}

RCM, RG 294.032, 25 anos, branca, natural e procedente de Patos de Minas, solteira. Foi encaminhada por facultativo com história de ter sido submetida a curetagem uterina convencional em 01/09/95 após suspeita clínica e ultrasonográfica de doença trofoblástica gestacional (30/08/95). O exame anátomo-patológico de $15 / 09 / 95$, veio a confirmar este suposto diagnóstico. Em 27/11/95 a paciente foi internada em nosso serviço, apresentando na radiografia de tórax metástase pulmonar no lobo médio do pulmão direito. Com este diagnóstico foi indicada quimioterapia com metotrexato (Mtx) $20 \mathrm{mg} /$ dia/ IM por 5 dias. A dosagem do $\beta$-hCG sangüíneo foi de $11.600 \mathrm{mUI} / \mathrm{ml}$. O quadro evolutivo das dosagens de $\beta$-hCG e as datas de quimioterapia são apresentados na Tabela 1.

Tabela 1 - Quadro evolutivo da paciente RCM após diagnóstico e tratamento da doença trofoblástica gestacional; datas e doses de quimioterapia e dosagens de $\beta$-hCG.

Data $\quad \begin{gathered}\text { Dosagem } \beta-\mathrm{hCG} \\ (\mathrm{mUI} / \mathrm{ml}) \text { sangüíneo }\end{gathered}$

\begin{tabular}{lcc}
$29 / 11 / 95$ & Mtx 20 mg/dia (5 dias) & 11.600 \\
20/12/95 & Mtx 20 mg/dia (5 dias) & 157 \\
$05 / 01 / 96$ & - & 27,6 \\
$17 / 01 / 96$ & Mtx 20 mg/dia (5 dias) & $<1$ \\
$06 / 02 / 96$ & Mtx 20 mg/dia (5 dias) & 12,6 \\
$26 / 02 / 96$ & Mtx 20 mg/dia (5 dias) & 2,6 \\
$13 / 03 / 96$ & - & 1,2 \\
$20 / 03 / 96$ & - & 1,9 \\
$08 / 04 / 96$ & - & 2,4 \\
\hline
\end{tabular}

Mtx - metotrexato.

Em 20/12/95, a paciente foi submetida ao $2^{\circ}$ ciclo de quimioterapia e a radiografia de tórax mantinha-se com presença de metástase pulmonar inalterada em relação ao exame anterior, embora houvesse decréscimo da dosagem sangüinea do $\beta$-hCG. Em 17/01/96, a paciente submeteu-se ao $3^{\circ}$ ciclo de quimioterapia, com dosagem do $\beta$-hCG de 27,6 $\mathrm{mUI} / \mathrm{ml}$, mas com persistência da imagem metastática pulmonar inalterada (Figura 1). No dia 06/02/96, iniciou-se o $4^{\circ}$ ciclo de quimioterapia mantendo-se inalterado o quadro de metástase pulmonar em radiografias realizadas em 06/02/96 e 26/02/96. Embora as dosagens de $\beta$-hCG continuassem a decrescer com negativação em 26/02/96, realizou-se o $5^{\circ}$ ciclo de quimioterapia. Em 13/03/96, submeteu-se à tomografia computadorizada de tórax, que demonstrou a presença de nódulo no lobo médio à direita (Figura 2), com a persistência da imagem pulmonar de metástase. Apesar da negativação das dosagens de $\beta$-hCG, foi indicada videotoracoscopia para exérese do nódulo em 12/04/96. A cirurgia transcorreu sem complicações. No dia 26/04/96, a paciente teve alta com radiografia normal (Figura 3). O resultado anátomo-patológico do nódulo foi de necrose, com ausência de neoplasia. Atualmente, a paciente continua em seguimento ambulatorial em bom estado geral com $\beta$-hCG negativo.

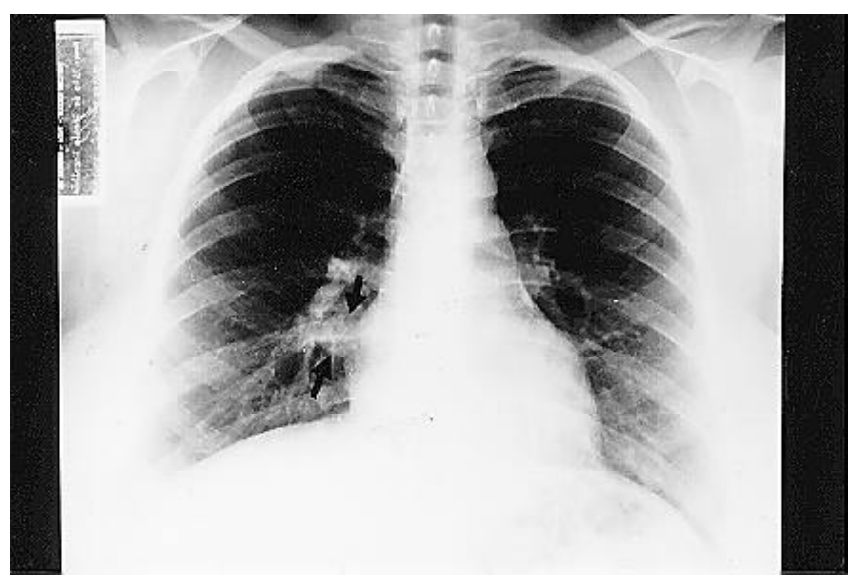

Figura 1 - Radiografia de tórax com presença de nódulo pulmonar metastático durante 0 tratamento com metotrexato.

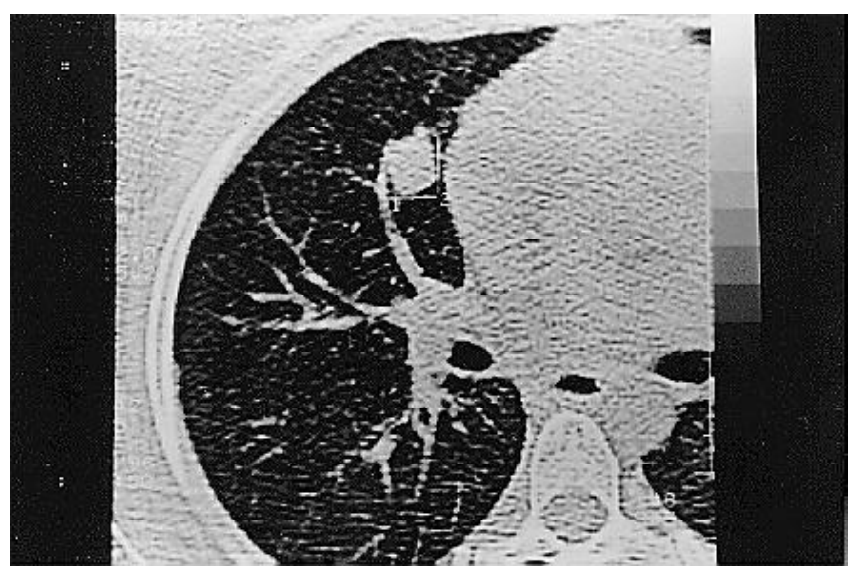

Figura 2 - Tomografia computadorizada apresentando nódulo pulmonar no lobo médio após cinco ciclos de quimioterapia. 


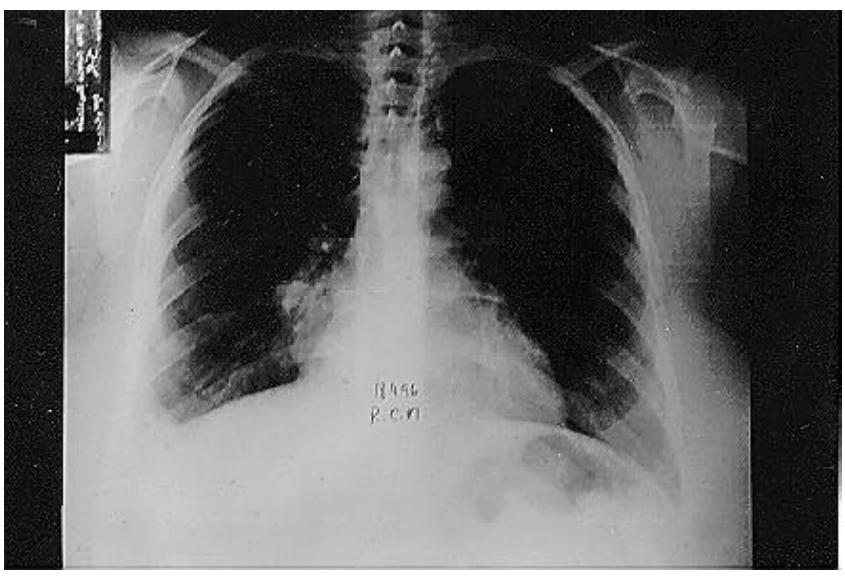

Figura 3 - Radiografia de tórax normal após a exérese por toracoscopia vídeo-assistida.

\section{Discussão}

Segundo Libshitz et al. ${ }^{6}$, existem três formas básicas de metástases pulmonares de DTG. Estes autores classificaram-nas em: a) típica - é a forma mais comum de lesão metastática descrita na literatura. É caracterizada por nódulos densos de contornos bem definidos semelhantes a metástases pulmonares de outros sítios primários. Geralmente são nódulos múltiplos e bilaterais, mas podem também se apresentar como nódulo único. A presença de cavitação também já foi descrita ${ }^{4}$; b) as metástase alveolares aparecem como múltiplas opacificações de pequeno tamanho não bemdefinidas em todo o pulmão, podendo ser parecidas com processo inflamatório quando as lesões são mais extensas; c) as do tipo embólica são as menos freqüentes entre as metástases pulmonares, caracterizando-se por oclusões intra-arteriais pulmonares na DTG embólica. As alterações radiográficas sugestivas de tromboembolismo ocorrem quando a embolização é suficiente para causar hipertensão pulmonar com alterações cardiovasculares.

Estes três tipos de metástase são os mais comuns, entretanto, há outras ocorrências raras de manifestações pulmonares. Entre elas, o derrame pleural pode estar presente e é devido à presença de nódulo pulmonar superficial com posterior sangramento para o espaço pleural ou a metástases pleurais ${ }^{9}$. Outra manifestação é a calcificação de um nódulo metastático após o tratamento com quimioterapia ${ }^{2}$. Everts et al. ${ }^{5}$ descreveram alterações pulmonares radiográficas caracterizadas por espessamentos de septos interlobulares, pequenos ou grandes nódulos e broncograma aéreo, achado clínico de febre, tosse não-produtiva, dispnéia grave e, às vezes, cianose. Estas alterações, segundo os autores, são provavelmente decorrentes da hipersensibilidade ao metotrexato que pode ocorrer em algumas pacientes. E, por fim, há relatos na literatura de persistência de nódulos na imagem radiológica do tórax, sendo que, após a resecção, encontrou-se fibrose ou necrose no exame histológico ${ }^{8,9}$, como no presente caso.

Neste relato, após a negativação do $\beta-\mathrm{hCG}$, as imagens pulmonares permaneceram, o que nos levou à indicação da exérese. A ressecção vídeoassistida de nódulos pulmonares periféricos está associada a menor trauma, recuperação funcional mais rápida e menor tempo de hospitalização do que a toracotomia convencional ${ }^{7}$. A maior desvantagem é a de não se poder contar com a palpação para identificar nódulos mais profundamente dispostos e eventualmente não identificados em exames de imagens préoperatórios. A análise histológica demonstrou necrose sem sinais de presença de células tumorais. Não obstante, o diagnóstico de certeza ser dado apenas pelo exame histológico, Swett \& Werlcott ${ }^{8}$ propõem que estes nódulos residuais podem ser considerados benignos se os títulos de $\beta$-hCG estiverem e permanecerem normais e se não houver crescimento destes nódulos no seguimento radiológico. A identificação deste tipo de alteração, embora rara, é de muita importância para persistência no tratamento oncológico clínico e/ ou cirúrgico.

\section{SUMMARY}

The aim of this report is to present one case of gestational trophoblastic disease with pulmonary metastases apparently persisting despite the return of $\beta$-human chorionic gonadotropin $(\beta-h C G)$ to normal levels after five cycles of chemotherapy (20 mg methotrexate/day for 5 days). The patient was submitted to a video-assisted thoracoscopy and the nodules were excised. Histological examination showed tissue necrosis without evidence of residual tumor. It is important to recognize that persistent nodules in the lungs of patients with metastatic gestational disease after treatment and normal $\beta$-hCG titers may not represent viable tumor but rather necrosis and/or fibrosis.

KEY WORDS: Trophoblastic gestational disease. Metastasis. Chemotherapy. Chorionic gonadotropin. 


\section{Referências}

1. Andrade JM, Murta EFC, Freitas MMS, Pires CR, Bighetti S. Mono and polichemotherapy in the treatment of metastatic and invasive gestational trophoblastic disease: Analysis of 50 cases. Rev Paul Med 1993; 111:433-438.

2. Cockshott WP, Hendrickse JP. Pulmonary calcification at the site of trophoblastic metastases. Br J Radiol 1969; 42:17-20.

3. De Baz PB, Lewis TJ. Imaging of gestational trophoblastic disease. Semin Oncol 1995; 22:130141.

4. Evans KT, Cockshott WP, Hendrickse JP. Pulmonary changes in malignant trophoblastic disease. $\mathrm{Br} \mathrm{J}$
Radiol 1965; 38:161-171.

5. Everts CS, Westcott JL, Bragg DG. Methotrexate therapy and pulmonary disease. Radiology 1973; 107:539-543.

6. Libshitz HI, Baber CE, Hammond CB. The pulmonary metastases of choriocarcinoma. Obstet Gynecol 1977; 49 (4):412-416.

7. Liu HP, Lin PJ, Hsieh MJ, Chang CH. Application of thoracoscopy for lung metastases. Chest 1995; 107:266- 268

8. Swett HA, Westcott JL. Residual nommalignant pulmonary nodules in choriocarcinoma. Chest $1974 ; 65: 560-562$.

9. Tow SH. The pulmonary lesion in choriocarcinoma. Proc R Soc Med 1967; 60:239-240.

\section{8음 CONGRESSO BRASILEIRO DE GINECOLOGIA E OBSTETRÍCIA}
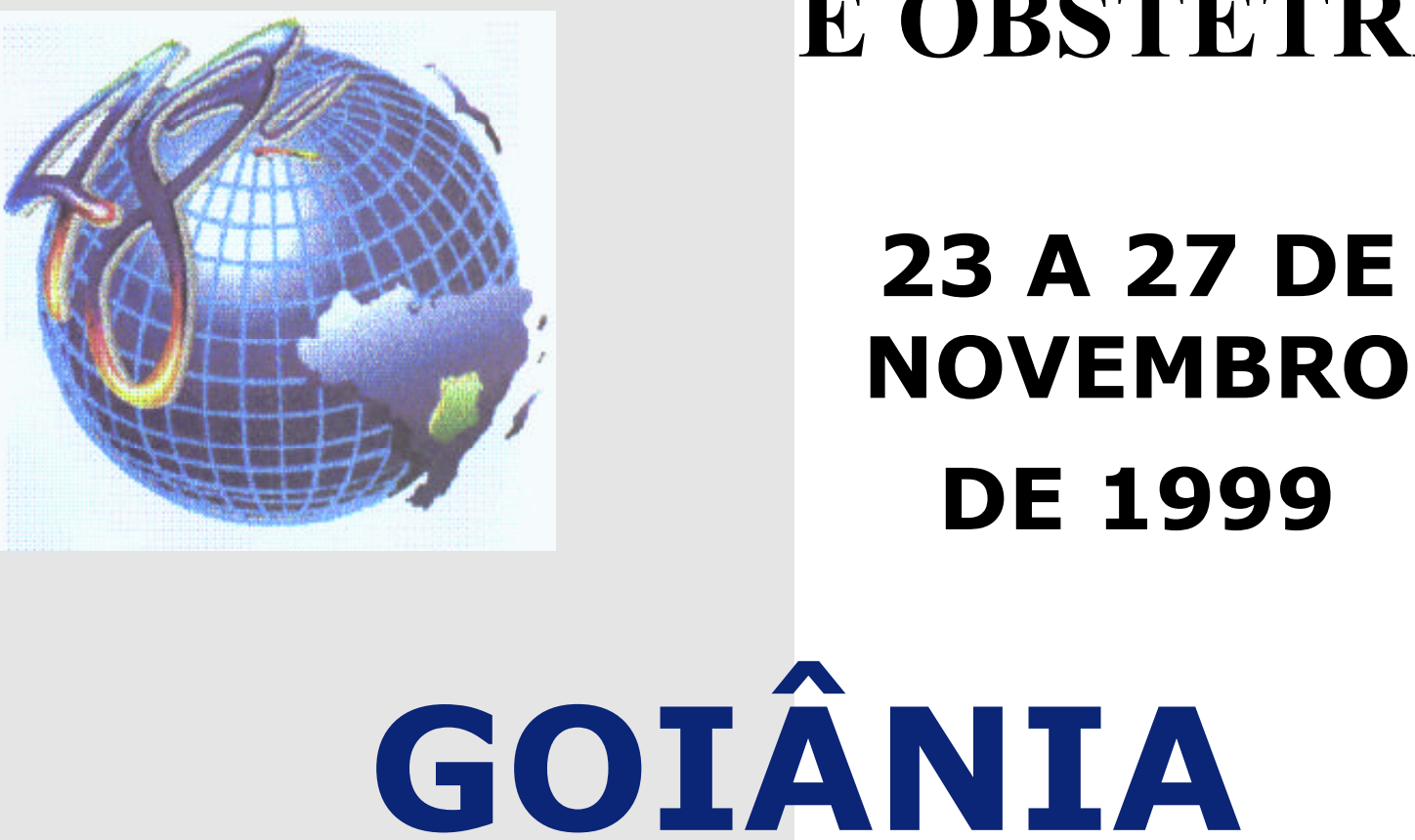

INFORMAÇÕES:

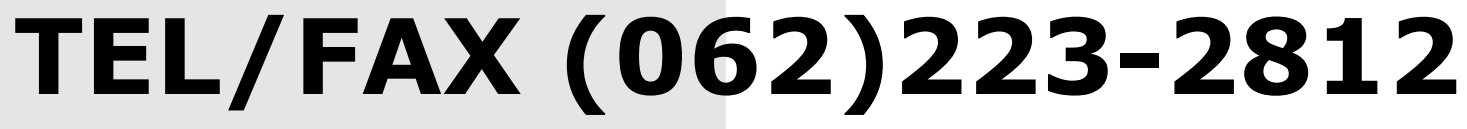

